\title{
Exact Averaging of Stochastic Equations for Flow in Porous Media
}

\author{
Mark Shvidler and Kenzi Karasaki
}

Earth Sciences Division, Lawrence Berkeley National Laboratory, 1 Cyclotron Rd, MS 901116, Berkeley, CA 94720; Mshvidler@lbl.gov, kkarasaki@lbl.gov

\begin{abstract}
It is well known that at present, exact averaging of the equations for flow and transport in random porous media have been proposed for limited special fields. Moreover, approximate averaging methods-for example, the convergence behavior and the accuracy of truncated perturbation series - are not well studied, and in addition, calculation of high-order perturbations is very complicated. These problems have for a long time stimulated attempts to find the answer to the question: Are there in existence some, exact, and sufficiently general forms of averaged equations? Here, we present an approach for finding the general exactly averaged system of basic equations for steady flow with sources in unbounded stochastically homogeneous fields. We do this by using (1) the existence and some general properties of Green's functions for the appropriate stochastic problem, and (2) some information about the random field of conductivity. This approach enables us to find the form of the averaged equations without directly solving the stochastic equations or using the usual assumption regarding any small parameters. In the common case of a stochastically homogeneous conductivity field we present the exactly averaged new basic nonlocal equation with a unique kernel-vector. We show that in the case of some type of global symmetry (isotropy, transversal isotropy, or orthotropy), we can for threedimensional and two-dimensional flow in the same way derive the exact averaged nonlocal equations with a unique kernel-tensor. When global symmetry does not exist, the nonlocal equation with a kernel-tensor involves complications and leads to an ill-posed problem.
\end{abstract}

Key words: heterogeneous porous media, random, flow, exact, averaging, nonlocal

\section{INTRODUCTION}

Recently, methods for analyzing flow and transport in random media have been finding everwidening applications. An effective description of flow and transport in irregular porous media entails interpreting permeability or conductivity fields as random functions of spatial coordinates, and flow velocity as a random function of spatial coordinates and time. Such a description also involves averaging of the stochastic system of flow and transport equations containing these functions (conservation laws, Darcy's law, and closing relations). The averaging problem involves finding the relationship between the nonrandom functionals of the unknown and the given fields - means, variations, distributions, densities, etc. — or a closed set of equations that contain these functionals. A certain interest attaches to the equations for the averaged functionals that are the laws of conservation of mass, momentum, and energy, which are invariant with respect to some set of conditions that uniquely determine the process (for example, the initial and boundary conditions). This is fundamentally possible, for example, in cases where the length scales of heterogeneity are extremely small. In physics and mathematical literature, this phenomenon is sometimes referred to as self-averaging (Lifshitz et al., 1998) and homogenization (Zhikov et al., 1994)

It is apparent that (in general) this is impossible, because in practical situations the process depends on a set of parameters that are usually not small, and thus an averaged description is 
used in computing the nonrandom characteristics (functionals) of random flow and transport processes for estimating the uncertainty of the processes. In some cases it is possible to find for averaged fields a closed system of equations.

Usually, the methods of averaging are approximate. Every so often, we can use a series expansion of small parameters, which (for example) specifies the deviation of some given fields from their mean values. Although it is possible to achieve results using this or a similar approach, it should be pointed out that the convergence of the perturbation expansion is insufficiently studied (Bonita and Cushman, 2001). For this reason, even if we can write a full perturbation expansion, there still exists the open question: Is a similar expansion the exact solution of an appropriate problem? Unfortunately, the answer to this question is usually absent.

Recently, there have been some studies in which the perturbation technique in frequency (Fourier) space was extensively used (King, 1987; Indelman and Abramovich, 1994; Indelman, 1996, 2001). In all these works authors reduce the problem for differential equations to second kind integral equations using the Fourier transformation (FT) of random Green function (King, 1987) or FT of head (Indelman and Abramovich, 1994). Note that these integral equations are invalid unless the generalized FT had been used, because the classical Fourier transformations of head and stochastically homogeneous fluctuation of conductivity do not exist. Next they formally apply the Liouville-Neuman iteration method to the integral equations. It is clear that the kernels and the free terms in the corresponding integral equations are not square integrable. Therefore, their iteration (perturbation) series always diverge. Nevertheless, using the leading terms of the series (Indelman and Abramovich) or a special partial summing of the diagrammatic series (King) under certain conditions can be valid for finding an approximation. In any case these approaches do not lead to exact results.

One effective approach is to utilize a distinctive space scale for fast oscillating fields as a small parameter. This approach, a so-called homogenization, was largely developed for investigating many linear and some nonlinear processes in periodical and random structures, which can be found in many publications (see, for example, books by Bakhvalov and Panasenko, 1984; Zhikov et al., 1994; Hornung, 1997). It should be noted that the exact effective conductivities formulas for a small number of special fields: stratified systems and some twodimensional systems with special symmetry are well known (Keller, 1964; Matheron, 1967; Dykhne, 1970). The exact formulas of effective conductivity for a three-dimensional case are not known.

It should be pointed out that there exist many problems that do not contain parameters naturally considered as small. In this general case, it is possible that significant mutual correlations of velocity and head (pressure) with the conductivity field exist at finite distances. To reflect this phenomenon in the averaged differential equations, there must be higher-order derivatives of the averaged field; that is, the averaged equations must be nonlocal.

Nonlocal averaged equations of flow have been suggested earlier. In some cases they are more-or-less hypothetical (Saffman, 1971; Dagan, 1979). Approximate nonlocal equations have been developed in the framework of perturbation methods (Lomakin, 1970; Shvidler, 1985; Keller, 2001).

Neuman and Orr (1993) considered steady flow in a bounded domain and found the averaged exact system of two equations. The first differential equation describes the auxiliary non-random field in a medium with the mean conductivity, and the second, almost-local, equation contains the desired mean field and the integral term with an auxiliary field. Thus, if the 
first local equation is solved and we know the auxiliary field, the second is a local differential equation, which is nonhomogeneous.

Here we note the significance of P. Indelman`s contribution in developing an original approach for analyzing flow and transport in random media, specifically, for nonlocal averaged equations (some of his publications can be found in the references at the end of this paper).

Generally speaking, direct averaging, as well as defining the functionals and the relationships between them, is exceptionally complicated. Later in this paper, we will show the forms for the exact relations between averaged fields, in some cases without actually solving the stochastic equations. We present a method for finding the general form of exactly averaged equations by using (1) some general properties of existing Green's functions for appropriate stochastic problems and (2) some information about the random fields of the conductivity. We present a new unique general form of the exactly averaged nonlocal equations for steady flow in threedimensional stochastically homogeneous random porous media with sources. We discuss the problem of uniqueness and the properties of the nonlocal averaged equations for the threedimensional and two-dimensional fields with some type of global symmetry (isotropic, transversally isotropic, and orthotropic). The determination of equations for mean fields is related to so-called inverse problems, which are often ill-posed. In our case, we have examples of uniqueness and non-uniqueness solutions for similar problems. In the present paper, we further develop the approach and some of the results that were presented briefly by Shvidler and Karasaki (1999, 2001, 2005), where in addition to steady flow we analyzed nonsteady transient flow and nonreactive solute transport.

2.Steady flow with sources and sinks in AN unbounded domain

We consider steady flow with sources and sinks that are locally or continuously distributed in a single-connected heterogeneous d-dimensional, porous, unbounded domain. The local condition of flow continuity and Darcy's law are given by the following equations:

$$
\nabla \mathbf{v}(\mathbf{x})=f(\mathbf{x}), \mathbf{v}(\mathbf{x})=\boldsymbol{\sigma}(\mathbf{x}) \mathbf{h}(\mathbf{x}), \mathbf{h}(\mathbf{x})=-\nabla u(\mathbf{x})
$$

Here, $\mathbf{x}=\left(x_{1}, \ldots, x_{d}\right)$ is a d-dimensional vector, $\mathbf{v}(\mathbf{x})$ is the Darcy's velocity vector, $\boldsymbol{\sigma}(\mathbf{x})$ is the conductivity tensor, the vector $\mathbf{h}(\mathbf{x})$ is hydraulic field intensity, and $u(\mathbf{x})$ is reduced pressure (or hydraulic head). The function $f(\mathbf{x})$ is the given density of sources and sinks, which is an integrable and compactly supported function or a distribution with bounded support. In this case, $q=\left|\int_{-\infty}^{\infty} f(\mathbf{x}) d x^{d}\right|$ is finite. Because the $\mathbf{h}(\mathbf{x})$ is a potential vector, we have the relation:

$$
\nabla \times \mathbf{h}(\mathbf{x})=0
$$

When $u(\mathbf{x})$ is the reduced pressure, $p^{*}(\mathbf{x})=p(\mathbf{x})-\rho \mathbf{g x}$, the conductivity is $\boldsymbol{\sigma}(\mathbf{x})=\mathbf{k}(\mathbf{x}) / \mu$; when $u(\mathbf{x})$ is the head, $p^{*}(\mathbf{x}) / \rho g$, the conductivity is $\boldsymbol{\sigma}(\mathbf{x})=\mathbf{k}(\mathbf{x}) \rho g / \mu$. Here, $p(\mathbf{x})$ is pressure, $\mathbf{k}(\mathbf{x})$ is the permeability tensor, $\mu=$ const and $\rho$ are liquid viscosity and density, respectively, and $\mathbf{g}$ is the acceleration of gravity vector.

We assume that conductivity $\boldsymbol{\sigma}(\mathbf{x})=\left\{\sigma_{l m}(\mathbf{x})\right\}$ is the second-rank tensor symmetric by subscripts and is a positive definite and limited local tensor. In this case, a unique symmetric and 
positive definite limited tensor $\mathbf{r}(\mathbf{x})=\boldsymbol{\sigma}^{-1}(\mathbf{x})$ exists, and we can write the conservative form of Darcy's law as a condition for momentum balance: $\mathbf{r}(\mathbf{x}) \mathbf{v}(\mathbf{x})=\mathbf{h}(\mathbf{x})$.

Note that the above-postulated symmetry of the tensors $\sigma_{l m}(\mathbf{x})$ and $r_{m l}(\mathbf{x})$ over the subscripts is natural. Clearly, adding an arbitrary skew-symmetric (over the subscripts) tensors to them does not impact the local dissipation of energy $e(\mathbf{x})=\mathbf{v}(\mathbf{x}) \mathbf{h}(\mathbf{x})=\mathbf{h}(\mathbf{x}) \boldsymbol{\sigma}(\mathbf{x}) \mathbf{h}(\mathbf{x})$. As shown by Zhikov et al. (1993), the symmetric tensor $\sigma_{l m}(\mathbf{x})$ is uniquely defined from an elliptic second-order operator $\nabla[\boldsymbol{\sigma}(\mathbf{x}) \mathbf{h}(\mathbf{x})]$. If the given tensor $\delta_{l_{m}}(\mathbf{x})$ is nonsymmetric, we will perform a regularization and use its symmetric part $\sigma_{l m}(\mathbf{x})=\left[\delta_{m}(\mathbf{x})+\delta \%_{m l}(\mathbf{x})\right] / 2$.

We use Equations (1) and the conditions for the function $f(\mathbf{x})$ to derive some estimations for the functions $\mathbf{v}(\mathbf{x}), \mathbf{h}(\mathbf{x})$ and $u(\mathbf{x})$ for large $|\mathbf{x}|$. Of course, the conditions for the solution at infinity must be consistent with the equations - that is, they must be consistent with the estimations. In addition, we will later use the estimations of random functions by Fourier transformation of some averaged functions.

For example, if $d=3$, the functions $\mathbf{v}(\mathbf{x})$ and $\mathbf{h}(\mathbf{x})$ vanish at infinity. The function $u(\mathbf{x})$ for $|\mathbf{x}| \rightarrow \infty$ can be an arbitrary constant and without a loss of generality, this constant can be defined as zero. These conditions at infinity ensure a unique solution for the system of Equations (1) in three-dimensional unbounded space.

In the two-dimensional case $(d=2)$, the estimation shows that if $q \neq 0$ and $|\mathbf{x}| \rightarrow \infty$, then $\mathbf{v}(\mathbf{x}) \rightarrow 0$ and $\mathbf{h}(\mathbf{x}) \rightarrow 0$, but $|u(\mathbf{x})| \rightarrow \infty$. The solution for the system of Equations (1), (2) is not unique. On the other hand, we can study the system of the first two equations from Equations (1) and Equation (2). This system is closed with respect to the vector-functions $\mathbf{v}(\mathbf{x})$ and $\mathbf{h}(\mathbf{x})$. These functions vanish at infinity, and the solution of the system is unique.

Note that if $f(\mathbf{x}) \neq 0$ but $q=0$, the vector-functions $\mathbf{v}(\mathbf{x}), \mathbf{h}(\mathbf{x})$ would tend to zero at infinity for $d=2,3$. In these two cases, without a loss of generality, we can write $u(\mathbf{x})=0$ for $x \rightarrow \infty$ and the system of Equations (1) has a unique solution.

Next we consider in detail a three-dimensional flow. We will return to the steady flow in two-dimensional space in Section 7.

\section{STOCHASTIC FORMULATION}

Let as assume that the tensor $\boldsymbol{\sigma}(\mathbf{x})$ is a stochastically homogeneous random field. That is, for any vector $\mathbf{x}$ and for an arbitrary vector $\mathbf{a}$, the finite-dimensional probability distributions for the random field $\boldsymbol{\sigma}(\mathbf{x}+\mathbf{a})$ does not depend on the arbitrary vector $\mathbf{a}$. Let $f(\mathbf{x})$ be a given, nonrandom density function. We introduce a unique random Green`s scalar function $g(\mathbf{x}, \mathbf{y})$, unique Green`s velocity vector functions $\gamma(\mathbf{x}, \mathbf{y})$ and intensity vector $\mathbf{s}(\mathbf{x}, \mathbf{y})$, for the problem described in Equations (1), so that for almost all realizations of the differentiable field $\boldsymbol{\sigma}(\mathbf{x})$, the functions $g(\mathbf{x}, \mathbf{y}), \gamma(\mathbf{x}, \mathbf{y})$ vanish at infinity, and satisfy the following equations: 


$$
\nabla_{x} \gamma(\mathbf{x}, \mathbf{y})=\delta(\mathbf{x}-\mathbf{y}), \gamma(\mathbf{x}, \mathbf{y})=\boldsymbol{\sigma}(\mathbf{x}) \mathbf{s}(\mathbf{x}, \mathbf{y}), \mathbf{s}(\mathbf{x}, \mathbf{y})=-\nabla_{x} g(\mathbf{x}, \mathbf{y})
$$

For the case in which the conductivity tensor components $\sigma_{l m}(\mathbf{x})$ are piecewise smooth functions, Green`s function $g(\mathbf{x}, \mathbf{y})$ must be a so-called weak solution of Equation (3) that satisfies the integral identity $\int \sigma_{l m}(\mathbf{x}) \nabla_{x} g(\mathbf{x}, \mathbf{y}) \nabla \varphi(\mathbf{x}) d x^{3}=\varphi(\mathbf{y})$, where $\varphi(\mathbf{x})$ is any arbitrary testing function that is infinitely differentiable and tends to zero at infinity. It is well known (see, for example, Bakhvalov and Panasenko, 1989) that the generalized solution $g(\mathbf{x}, \mathbf{y})$ vanishes at infinity and satisfies the Equations (3) at all points where $\boldsymbol{\sigma}(\mathbf{x})$ is smooth. On the surfaces where tensor $\boldsymbol{\sigma}(\mathbf{x})$ is disconnected, the function $g(\mathbf{x}, \mathbf{y})$ and normal component of velocity-vector $\gamma(\mathbf{x}, \mathbf{y})$ are uninterrupted.

Now we can convey the solution for the problem of Equations (1) and (2) through density function $f(\mathbf{x})$ and the random solution of the system of Equations (3), and write:

$$
u(\mathbf{x})=\int g(\mathbf{x}, \mathbf{y}) f(\mathbf{y}) d y^{3}, \mathbf{h}(\mathbf{x})=\int \mathbf{s}(\mathbf{x}, \mathbf{y}) f(\mathbf{y}) d y^{3}, \mathbf{v}(\mathbf{x})=\int \boldsymbol{\gamma}(\mathbf{x}, \mathbf{y}) f(\mathbf{y}) d y^{3}
$$

where the integration is over the entire unbounded 3-D space.

Note that although the functions $g(\mathbf{x}, \mathbf{y}), \mathbf{s}(\mathbf{x}, \mathbf{y})$ and $\gamma(\mathbf{x}, \mathbf{y})$ are integrable in any bounded domain in $R^{3}$ and vanish at infinity, they are not integrable in a full three-dimensional space. But the integrals in Equations (4) make sense, because the function $f(\mathbf{x})$ is compactly supported.

We also introduce the averaged fields over the ensemble of realizations of the random $\boldsymbol{\sigma}(\mathbf{x}): U=\langle u\rangle, \mathbf{V}=\langle\mathbf{v}\rangle, \mathbf{H}=\langle\mathbf{h}\rangle, G=\langle g\rangle, \mathbf{S}=\langle\mathbf{s}\rangle, \boldsymbol{\Gamma}=\langle\gamma\rangle$. As long as $\boldsymbol{\sigma}(\mathbf{x})$ is a stochastically homogeneous field, the mean Green's function $G(\mathbf{x}, \mathbf{y})$, the mean Green`s velocity $\Gamma(\mathbf{x}, \mathbf{y})$, and mean hydraulic intensity $\mathbf{S}(\mathbf{x}, \mathbf{y})$ are invariant over translation in space, and therefore depend only on the difference $\mathbf{x}-\mathbf{y}$. Because the local operator $\nabla_{x}\left[\boldsymbol{\sigma}(\mathbf{x}) \nabla_{x} g(\mathbf{x}, \mathbf{y})\right]$ is self-adjoint, Green`s function $g(\mathbf{x}, \mathbf{y})$ is symmetric - that is, it satisfies the reciprocity law (Courant, 1962): $g(\mathbf{x}, \mathbf{y})=g(\mathbf{y}, \mathbf{x})$ and likewise, $G(\mathbf{x}, \mathbf{y})=G(\mathbf{y}, \mathbf{x}), G(\mathbf{x}-\mathbf{y})=G(\mathbf{y}-\mathbf{x})$. Hence, $G(\mathbf{x}-\mathbf{y})$ is a real, even function, the function $\mathbf{S}(\mathbf{x}-\mathbf{y})$ is a real, odd vector.

Averaging the Equations (4) over the ensemble, we have:

$$
\begin{aligned}
& U(\mathbf{x})=\int G(\mathbf{x}-\mathbf{y}) f(\mathbf{y}) d y^{3}, \mathbf{H}(\mathbf{x})=\int \mathbf{S}(\mathbf{x}-\mathbf{y}) f(\mathbf{y}) d y^{3}=-\nabla U(\mathbf{x}) \\
& \mathbf{V}(\mathbf{x})=\int \boldsymbol{\Gamma}(\mathbf{x}-\mathbf{y}) f(\mathbf{y}) d y^{3}, \quad \boldsymbol{\Gamma}(\mathbf{x}-\mathbf{y})=\langle\boldsymbol{\sigma}(\mathbf{x}) \mathbf{s}(\mathbf{x}, \mathbf{y})\rangle=-\left\langle\boldsymbol{\sigma}(\mathbf{x}) \nabla_{x} g(\mathbf{x}, \mathbf{y})\right\rangle
\end{aligned}
$$

and after averaging the first equation from (3), we find the relationship of compatibility for the $\boldsymbol{\Gamma}(\mathbf{x}-\mathbf{y})$ components:

$$
\nabla_{x} \boldsymbol{\Gamma}(\mathbf{x}-\mathbf{y})=\delta(\mathbf{x}-\mathbf{y})
$$

Thus, the functions $U(\mathbf{x}), \mathbf{H}(\mathbf{x})$, and $\mathbf{V}(\mathbf{x})$ are presented as convolutions, which makes sense because the function $f(\mathbf{x})$ has bounded support. Finally, after averaging the first equation from (1), we have:

$$
\nabla \mathbf{V}(\mathbf{x})=f(\mathbf{x})
$$


Equations (5), (6), and (7) make up a system of equations for the averaged fields $U(\mathbf{x})$, $\mathbf{H}(\mathbf{x})$, and $\mathbf{V}(\mathbf{x})$. This system contains the kernels $G(\mathbf{x}-\mathbf{y}), \mathbf{S}(\mathbf{x}-\mathbf{y})$, and $\boldsymbol{\Gamma}(\mathbf{x}-\mathbf{y})$, which are nonrandom functionals of the random conductivity field $\boldsymbol{\sigma}(\mathbf{x})$ and the random Green's function $g(\mathbf{x}, \mathbf{y})$. Of course, explicit definitions of the functionals $G, \mathbf{S}$ and $\boldsymbol{\Gamma}$ are very difficult to obtain. For now, the existence of these functionals in itself is sufficient. It is possible to determine some of their features, which help in finding a general form for the averaged equations, of which Equations (5), (6), are a part. Later in this paper, we will find them in different forms.

\section{FOURIER ANALYSIS}

To analyze equations with convolutions in all space, we consider for absolute integrable in full space function $\psi(\mathbf{x})$ the classical Fourier transform $T_{F}[\psi(\mathbf{x})]=\bar{\psi}(\mathbf{k})$ and inverse Fourier transform $T_{F}^{-1}[\bar{\psi}(\mathbf{k})]=\psi(\mathbf{x})$ :

$$
\bar{\psi}(\mathbf{k})=\int \exp [-2 \pi i(\mathbf{x} \cdot \mathbf{k})] \psi(\mathbf{x}) d x^{3}, \psi(\mathbf{x})=\int \exp [2 \pi i(\mathbf{k} \cdot \mathbf{x})] \bar{\psi}(\mathbf{k}) d k^{3},
$$

It easy to show that the functions $G(\mathbf{x})$ and $U(\mathbf{x}), \mathbf{H}(\mathbf{x}), \Gamma(\mathbf{x})$, and $\mathbf{V}(\mathbf{x})$ slowly vanish at infinity, and therefore they are not integrable in full space. The integrals in (8) for these functions diverge, and the so-called generalized Fourier transformation of similar functions or distributions should be used - for example, see L. Schwartz (1961) and K. Yosida (1978).

The generalized Fourier transformation $\bar{T}_{F}$ of distribution (functional) $q$ over A-space testing functions $\varphi$ is a distribution (functional) $\bar{q}$ over B-space functions $\bar{\varphi}=\mathrm{T}_{F}[\varphi]$. So, for $\bar{q}=\overline{\mathrm{T}}_{F}[q]$ and $q=\overline{\mathrm{T}}_{F}^{-1}[\bar{q}]$, we have the equality for scalar products $\left(\bar{T}_{F}[q], \bar{\varphi}\right)=(q, \varphi)$ :

$$
\int \bar{q}^{*}(\mathbf{k}) \bar{\varphi}(\mathbf{k}) d k^{3}=\int q^{*}(\mathbf{x}) \varphi(\mathbf{x}) d x^{3}
$$

Here, the star-superscript indicates the complex conjugate.

It is known that Equation (9) is the definition of generalized direct and inverse Fourier transformations, and if $q(\mathbf{x})$ is an absolute integrable function, Equation (9) is equivalent to (8).

The commonly used testing function spaces $\varphi(\mathbf{x})$ are: (1) the so-called testing function of functional space $S_{\infty}$, which includes all infinitely differentiable functions that decrease more rapidly at infinity than any power of $1 /|\mathbf{x}| ;(2)$ the functions that belong to $\mathrm{K}$ functional space of infinitely differentiable functions with finite support.

A generalized Fourier transformation for arbitrary distribution does not exist. But definition (9) is valid for some special distributions (for example, so-called slow growth or tempered distributions). Any local integrable function that grows more rapidly at infinity than any power function is tempered. The generalized functions with bounded support are also tempered (Schwartz, 1961).

For the scalar functions $G(\mathbf{x}), U(\mathbf{x})$ and vector functions $\mathbf{S}(\mathbf{x}), \mathbf{H}(\mathbf{x}), \boldsymbol{\Gamma}(\mathbf{x}), \mathbf{V}(\mathbf{x})$, in Section 3 we write a system of equations, part of which contains convergent convolutions. Now we apply to all these equations the generalized Fourier transformation defined by Equation (9). Taking into account that the functions $G(\mathbf{x}), U(\mathbf{x})$, and $\Gamma(\mathbf{x})$ are tempered (they even decrease 
at infinity) and that the function $f(\mathbf{x})$ has bounded support, we can present the generalized Fourier transformation of the convolutions as a product of generalized Fourier transformations of appropriate functions. As a result, from Equations (5), (6), and (7), we can write the following system of linear algebraic equations in $\mathbf{k}$-space:

$$
\bar{U}(\mathbf{k})=\bar{G}(\mathbf{k}) \bar{f}(\mathbf{k}), \overline{\mathbf{H}}(\mathbf{k})=\overline{\mathbf{S}}(\mathbf{k}) \bar{f}(\mathbf{k})=2 \pi i \mathbf{k} \bar{U}(\mathbf{k}), \overline{\mathbf{V}}(\mathbf{k})=\overline{\mathbf{\Gamma}}(\mathbf{k}) \bar{f}(\mathbf{k})
$$

In addition, we can write, for $\bar{\Gamma}_{l}(\mathbf{k})$ and $\bar{V}_{l}(\mathbf{k})$, the conditions of compatibility:

$$
2 \pi i k_{l} \bar{\Gamma}_{l}(\mathbf{k})=1,2 \pi i k_{l} \bar{V}_{l}(\mathbf{k})=\bar{f}(\mathbf{k})
$$

If the functions $\bar{G}(\mathbf{k}), \bar{\Gamma}(\mathbf{k})$ and $\bar{f}(\mathbf{k})$ are known, the Equations (10)-(11) are a closed system with respect to functions $\bar{U}(\mathbf{k}), \overline{\mathbf{H}}(\mathbf{k})$, and $\overline{\mathbf{V}}(\mathbf{k})$.

After eliminating $\bar{f}(\mathbf{k})$ from the Equation (10) and Equation (11), we find the local equations that for each $\bar{f}(\mathbf{k})$ bind the scalar field $\bar{U}(\mathbf{k})$ and the vector field $\overline{\mathbf{V}}(\mathbf{k})$ :

$$
\bar{V}_{l}(\mathbf{k})=\bar{\Pi}_{l}(\mathbf{k}) \bar{U}(\mathbf{k}) \quad, \quad \bar{\Pi}_{l}(\mathbf{k})=\bar{\Gamma}_{l}(\mathbf{k})[\bar{G}(\mathbf{k})]^{-1}
$$

From (10), (11), and (12) we obtain for $\bar{\Pi}_{l}(\mathbf{k})$ the condition of compatibility:

$$
2 \pi i k_{l} \bar{\Pi}_{l}(\mathbf{k})=[\bar{G}(\mathbf{k})]^{-1}
$$

Equations (10), (11), and (12), (13) are also a closed basic system with respect to functions $\bar{U}(\mathbf{k})$ and $\overline{\mathbf{H}}(\mathbf{k}), \overline{\mathbf{S}}(\mathbf{k}), \overline{\mathbf{V}}(\mathbf{k})$.

It is evident that the vector $\overline{\mathbf{\Pi}}(\mathbf{k})$ is not dependent on $\bar{f}(\mathbf{k})$ : it is a unique operator that transforms the scalar field $\bar{U}(\mathbf{k})$ to the corresponding vector field $\overline{\mathbf{V}}(\mathbf{k})$. If the appropriate convolution converges, we can write a nonlocal equation with the kernel-vector $\Pi(\mathbf{\Pi}-\mathbf{y})$ :

$$
\mathbf{V}(\mathbf{x})=\int \boldsymbol{\Pi}(\mathbf{x}-\mathbf{y}) U(\mathbf{y}) d y^{3}
$$

The relations between the two vector fields $\overline{\mathbf{H}}(\mathbf{k})$ and $\overline{\mathbf{V}}(\mathbf{k})$ and (respectively) $\mathbf{H}(\mathbf{x})$ and $\mathbf{V}(\mathbf{x})$ will be analyzed later in Section 5 for the cases of special field symmetry, and in Section 6 for the general case. Here, we will only recall the well-known and evident fact that, in general, the set of operators that transform a given vector to another noncollinear vector is infinite.

Clearly, the exact averaged Equation (12) is reversible. If we know the scalar field $\bar{U}(\mathbf{k})$, from (12) we can directly define the vector field $\overline{\mathbf{V}}(\mathbf{k})$ and vice versa. If we know the field $\overline{\mathbf{V}}(\mathbf{k})$, from Equations (12) and (13) we can find $\bar{U}(\mathbf{k})=2 \pi i \bar{G}(\mathbf{k}) \mathbf{k} \overline{\mathbf{V}}(\mathbf{k})$.

Note that in the former analysis, we do not suppose the existence of any small parameters.

\section{GLOBAL SYMMETRY}

We continue the analysis of the averaged equations and assume that the multipoint moments of the conductivity random field $\boldsymbol{\sigma}(\mathbf{x})$ satisfy some symmetry conditions related to the structural properties of the field as a whole. We shall call this type of symmetry global. We will describe three types of global symmetry and designate them with initials: i (isotropy), o (orthotropy), and $\mathrm{t}$ (transversal isotropy). The isotropy case is presented in detail below, followed by a brief discussion of the other two. 
Let the random conductivity tensor $\boldsymbol{\sigma}(\mathbf{x})$ be a global isotropic field. In this case, the imaginary vector $\overline{\mathbf{\Pi}}(\mathbf{k})$ in any orthogonal coordinate system is proportional to the uniquely defined vector $2 \pi i \mathbf{k}$. The real proportionality scalar coefficient depends entirely on $k=|\mathbf{k}|$ and is invariant for any rotation and reflection on the coordinate planes $k_{l}=0$. We can write

$$
\bar{\Pi}_{l}^{(i)}(\mathbf{k})=-\bar{B}^{(i)}(k) 2 \pi i k_{l}
$$

Here $B^{(i)}(k)$ is a scalar and positive function. Then

$$
\bar{V}_{l}(\mathbf{k})=\overline{\mathrm{B}}_{l m}^{(i)}(k) \bar{H}_{m}(\mathbf{k}), \overline{\mathrm{B}}_{l m}^{(i)}(k)=B^{(i)}(k) \delta_{l m}, \bar{H}_{m}(\mathbf{k})=-2 \pi i k_{m} \bar{U}(\mathbf{k})
$$

and therefore in $x$-space, if appropriate convolutions converge, we have the relation:

$$
\mathbf{V}(\mathbf{x})=-\int \mathbf{B}^{(i)}(|\mathbf{x}-\mathbf{y}|) \nabla U(\mathbf{y}) d y^{3}
$$

Here $\mathbf{B}^{(i)}(|\mathbf{x}|)$ is a unique, real spherical tensor.

It is evident that Equation (16) is reversible and we can write:

$$
\bar{R}_{m l}^{(i)}(k) \bar{V}_{l}(\mathbf{k})=\bar{H}_{m}(\mathbf{k}), \bar{R}_{m l}^{(i)}(k)=\left[\bar{B}^{(i)}(k)\right]^{-1} \delta_{m l}
$$

In $x$-space we have the nonlocal condition of the mean momentum balance with unique kernel -isotropic resistance tensor $\mathbf{R}(|\mathbf{x}|)=\bar{T}_{F}^{-1}[\overline{\mathbf{R}}(|\mathbf{k}|)]$ :

$$
\int R_{m l}^{(i)}(|\mathbf{x}-\mathbf{y}|) V_{l}(\mathbf{y}) d y^{3}=H_{m}(\mathbf{x})
$$

Note that nonlocal equations (17) and (19) are meaningful only when appropriate convolutions converge. Here, we describe the case of global isotropic porous media and use the information on the asymptotic behavior of function $G(|\mathbf{x}|)$ for large and small $|\mathbf{x}|$. Inserting into Equation (13) the expression $\overline{\boldsymbol{\Pi}}(\mathbf{k})=-2 \pi j \overline{\mathbf{R}}^{-1}(k) \mathbf{k}$, we find $\bar{R}_{m l}(k)=4 \pi^{2} k^{2} \bar{G}(k) \delta_{m l}$ or we have $R_{m l}(x)=-\nabla^{2} G(x) \delta_{m l}$. Installing the last formula in Equation (19) yields: $H_{m}(\mathbf{x})=\int \nabla G(\mathbf{y}) \nabla_{y} V_{m}(\mathbf{x}-\mathbf{y}) d y^{3}$. If the field $\mathbf{V}(\mathbf{x}-\mathbf{y})$ is differentiable, the convergence of the convolution in (19) depends on the behavior of the integrand at $\mathbf{y}=0$ and $|\mathbf{y}| \rightarrow \infty$. An investigation of the asymptotic behaviors of the function $G(y)$ (Shvidler, 1966; 1985) showed that if scale heterogeneity is finite and $y$ is very small, the principal part of the Green`s function is $G(y) \sim 1 / 4 \pi \sigma_{*} y$. Here $\sigma_{*}=\left\langle\sigma^{-1}\right\rangle^{-1}$ is the mean harmonic conductivity. For very large $y$, it was also shown that $G(y) \sim 1 / 4 \pi \sigma^{*} y$, where $\sigma^{*}$ is a finite effective conductivity. It easy to find the estimate for gradients of flow velocity and Green`s function for very large and small $y$. We can find that the integral converges at $\mathbf{y}=0$ and at infinity, which means that convolution like the one in Equation (19) does exist.

If the field $\boldsymbol{\sigma}(\mathbf{x})$ is globally orthotropic, then there exists some orthogonal coordinate system such that all the stochastic multipoint moments of the random field are invariant to the reflection on the planes $x_{l}=0$. In this coordinate system, the vector $\boldsymbol{\Gamma}(\mathbf{x})$ is the product of the diagonal tensor that depends on $\left|x_{1}\right|,\left|x_{2}\right|$ and $\left|x_{3}\right|$ and the vector $\mathbf{x}$. Therefore, $\boldsymbol{\Gamma}(\mathbf{x})$ is an odd, 
real function and $\overline{\boldsymbol{\Gamma}}(\mathbf{k})$ and $\overline{\boldsymbol{\Pi}}(\mathbf{k})$ are odd and imaginary vector functions. In general they are noncollinear with the vector $\mathbf{k}$. In this case, the components $\bar{\Pi}_{l}(\mathbf{k})$ can be written:

$$
\bar{\Pi}_{l}^{(o)}(\mathbf{k})=-\bar{B}_{l}^{(o)}(\mathbf{k}) 2 \pi i k_{l},(\text { summation over } l \text { is not implied!) }
$$

The three functions $\bar{B}_{l}^{(o)}(\mathbf{k})$ are positive, even, and depend on $\left|k_{1}\right|,\left|k_{2}\right|,\left|k_{3}\right|$.

If the field $\boldsymbol{\sigma}(\mathbf{x})$ is globally transversal isotropic, then we can write

$$
\bar{\Pi}_{l}^{(t)}(\mathbf{k})=B_{l}^{(t)}(\mathbf{k}) 2 \pi i k_{l},(\text { no summation over } l !)
$$

The three functions $\bar{B}_{l}^{(t)}(\mathbf{k})$ are invariant relative to the rotation around only one axis of the coordinate system (for example, $k_{3}$ ) and reflection on any planes $k_{l}=0$. They are positive, even, and depend on $\left(k_{1}^{2}+k_{2}^{2}\right)^{1 / 2}$ and $\left|k_{3}\right|$.

Thus, in all three basic cases of symmetry $(\alpha=i, t, o)$ with a suitable orientation of the coordinate axes, (bear in mind that in each of the cases, the diagonal tensors $\overline{\mathbf{B}}^{(\alpha)}(\mathbf{k})$ remain invariant in relation to superscript $\alpha)$, the averaged equation is

$$
\overline{\mathbf{V}}(\mathbf{k})=\overline{\mathrm{B}}^{(\alpha)}(\mathbf{k}) \overline{\mathbf{H}}(\mathbf{k}), \overline{\mathrm{B}}_{l m}^{(\alpha)}(\mathbf{k})=\delta_{l m} \bar{B}_{m}^{(\alpha)}(\mathbf{k}) \text {, (no summations over } m ! \text { ) }
$$

Equation (22) is reversible, and for any $\alpha$ we have

$$
\overline{\mathbf{R}}^{(\alpha)}(\mathbf{k}) \overline{\mathbf{V}}(\mathbf{k})=\overline{\mathbf{H}}(\mathbf{k}), \quad \bar{R}_{m l}^{(\alpha)}(\mathbf{k})=\delta_{m l}\left[\bar{B}_{m}^{(\alpha)}(\mathbf{k})\right]^{-1}
$$

In $x$-space in a corresponding coordinate system, if appropriate convolutions converge, we have the nonlocal equations with unique kernels-diagonal tensors $\mathbf{B}^{(\alpha)}(\mathbf{x}-\mathbf{y})$ and $\mathbf{R}^{(\alpha)}(\mathbf{x}-\mathbf{y})$, that are invariant relative $(\alpha)$-kind transformation:

$$
\mathbf{V}(\mathbf{x})=-\int \mathbf{B}^{(\alpha)}(\mathbf{x}-\mathbf{y}) \nabla U(\mathbf{y}) d y^{3}, \int \mathbf{R}^{(\alpha)}(\mathbf{x}-\mathbf{y}) \mathbf{V}(\mathbf{y}) d y^{3}=-\nabla U(\mathbf{x})
$$

Now we consider the diagonal tensors $\overline{\mathbf{B}}^{(\alpha)}(\mathbf{k})$ and $\mathbf{B}^{(\alpha)}(\mathbf{x})$ with real and even components and write the component $\bar{B}_{l l}^{(\alpha)}(\mathbf{k})$ in the following form:

$$
\overline{\mathrm{B}}_{l l}^{(\alpha)}(\mathbf{k})=\hat{\overline{\mathrm{B}}}_{l l}^{(\alpha)} \bar{F}_{l l}^{(\alpha)}(\mathbf{k})
$$

Here $\hat{\bar{B}}_{l l}^{(\alpha)}=\lim \bar{B}_{l l}^{(\alpha)}(\varepsilon \mathbf{k})$ where a positive dimensionless number $\varepsilon \rightarrow 0$. The variables

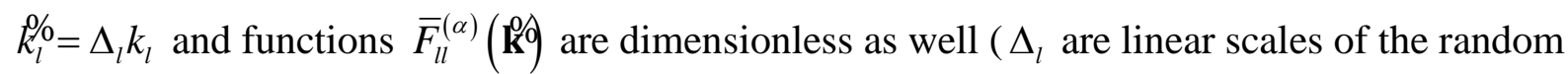
field $\boldsymbol{\sigma}(\mathbf{x})$, for example, the correlation scales). However, by the limit tendency for the homothetic transformation, the ratios between the scales of heterogeneity should be remain in the limit. Assuming the existence of a Taylor's expansion of the function $\bar{F}_{l l}^{(\alpha)}(\mathbf{k})$ around the point $\mathbf{k}=0$, we can write:

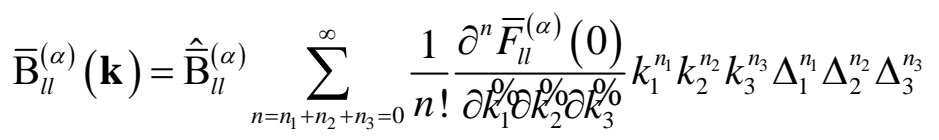

Substituting (26) into (22) and taking into account that all the odd derivatives of $\bar{F}_{l l}^{(\alpha)}(\mathbf{k})$ at $\mathbf{k}^{\circ}=0$ are zero, we can write the expansions for the mean velocity $V_{l}^{(\alpha)}(\mathbf{x})$ in $x$-space: 


$$
V_{l}(\mathbf{x})=-\hat{\overline{\mathrm{B}}}_{l l}^{(\alpha)} \sum_{n=n_{1}+n_{2}+n_{3}=0}^{\infty} \frac{\Delta_{1}^{2 n_{1}} \Delta_{2}^{2 n_{2}} \Delta_{3}^{2 n_{3}} \mathrm{I}_{l l, n}^{(\alpha)}\left(n_{1}, n_{2}, n_{3}\right)}{(2 n) !} \frac{\partial^{2 n+1} U(\mathbf{x})}{\partial x_{l} \partial x_{1}^{2 n_{1}} \partial x_{2}^{2 n_{2}} \partial x_{3}^{2 n_{3}}}
$$

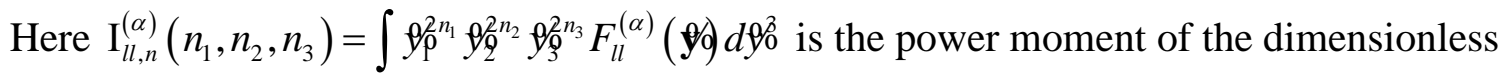
function $F_{l l}^{(\alpha)}(\emptyset)=T_{F}^{-1}\left[\bar{F}_{l l}^{(\alpha)}(\mathbf{k})\right]$ of the dimensionless variables $y / p=x_{l} / \Delta_{l}$. It is clear that from Equation (25), we have $\bar{F}_{l l}^{(\alpha)}(0)=1$ and then $\mathrm{I}_{l l, 0}^{(\alpha)}(0,0,0)=1$; the first term in the sum in (27) does not depend on $\Delta_{l}$ and is $\frac{\partial U(\mathbf{x})}{\partial x_{l}}$. We assume that for any $l$ and $m$ the ratio $\Delta_{l} / \Delta_{m}=$ const , which conserves the homothety by passage $\Delta_{l}$ to the vanishing limit. In the limiting case $\Delta_{l} \rightarrow 0$, all other terms in the summation (27) tend to zero, which corresponds with the theory of homogenization. In this limiting case, we have the averaged equation:

$$
\mathbf{V}(\mathbf{x})=-\hat{\overline{\mathbf{B}}}^{(\alpha)} \mathbf{H}(\mathbf{x}), \mathbf{H}(\mathbf{x})=-\nabla U(\mathbf{x})
$$

Here $\hat{\overline{\mathbf{B}}}^{(\alpha)}=$ const is the diagonal effective conductivity tensor.

Now we discuss under what conditions we can consider so-called homogenization in full space. We should compare the scales, for example, correlation scales of conductivity field, with other macro scales of the field and consider the conditions that describe flow in this field.

In the problem studied thus far, we have only one macro structure that have some finite length scale-it is the source density - that is a compactly supported and square integrable function, $f(\mathbf{x})$. If this function is sufficiently complicated it is natural to select plenty of scales. In particular, we must compare them against the scales of heterogeneity. It is prudent to formalize a procedure for finding small macro scales of the density function.

For example, let us expand the function $f(\mathbf{x})$ in the Fourier integral $\bar{f}(\mathbf{k})$. Having given some level of significance to $\mathscr{E} \varnothing 0$, we introduce function $\bar{f}_{\varepsilon_{0}}(\mathbf{k})=\bar{f}(\mathbf{k})$, if $|\bar{f}(\mathbf{k})|>\varepsilon$, and $\bar{f}_{\varepsilon_{0}}(\mathbf{k})=0$, if $|\bar{f}(\mathbf{k})| \leq \varepsilon$. Thus the function $\bar{f}_{\varepsilon_{0}}(\mathbf{k})$ is the contribution in the expansion of the oscillations that are at least $\varepsilon /$-significant. Now, select some largest "zero-border" frequency $\mid \mathbf{k}_{\theta d}$ we assume that $\Delta_{\varepsilon \circ}: 1 / \mid \mathbf{k}_{\varepsilon \ell}$ is least length scale of the density function $f(\mathbf{x})$. Finally we assume that in homogenization limit the scales of heterogeneity $\Delta_{l}$ should be: $\Delta_{l} / \Delta_{\mathscr{g}}=1$.

Notice that according to the theory of homogenization, the tensor of the effective conductivity exists and is constant in all Euclidian space $R^{3}$. This is true if, for example, in any limited domain $Q * \subset R^{3}$, the source density $f(\mathbf{x})$ is a square integrable function. Furthermore, if in any orthogonal coordinate system, the tensor of the local random conductivity is symmetric and positive definite, the tensor of the effective conductivity is also symmetric and positive definite, so-called elliptic (Zhikov et al., 1993). Thus, the principal part of the expansion in Equation (27) corresponds with the theory of homogenization limit and can be used for computing the effective conductivity. Clearly, the principal axes for all the tensors $\overline{\mathbf{B}}^{(\alpha)}(\mathbf{k})$ and $\overline{\mathbf{R}}^{(\alpha)}(\mathbf{k})$ for any $\mathbf{k}$, for each $\alpha$, are identical to the respective coordinate axes.

Here we point out that the Indelman and Abramovich (1994) paper contains equations-(15) and (16) in their paper - that resemble the first equation in (28) of our paper. However, they are 
not equivalent; in fact they are dissimilar. In the first place, as we discussed in Section 1, the variant of the perturbation method developed by Indelman and Abramovich (1994) is unusable for finding the exact solution. Next, the definition of effective conductivity used by them-the conductivity of stochastically homogeneous media for the mean flow, which is uniform in full space_-cannot work for any dimensional unbounded media, because the uniform flow in such media does not exist.

Up to this point, we have studied the fields with some symmetry in special orthogonal coordinate systems. If the orthogonal coordinate axes $x_{l}^{\prime}$ and $k_{l}^{\prime}$ are oriented arbitrarily, and $\beta_{l m}$ is the cosine of the angle between the axes $x_{l}^{\prime}$ and $x_{m}$, the effective conductivity tensor in the new coordinate system is $\overline{\mathbf{B}}^{\prime(\alpha)}=\boldsymbol{\beta} \overline{\mathbf{B}}^{(\alpha)} \boldsymbol{\beta}^{-1}$. This tensor is symmetric and positive definite (elliptic). The averaged equations in the arbitrary coordinate system $x_{l}^{\prime}$ have the forms $\mathbf{V}^{\prime}\left(\mathbf{x}^{\prime}\right)=-\overline{\mathbf{B}}^{\prime(\alpha)} \nabla U^{\prime}\left(\mathbf{x}^{\prime}\right), \overline{\mathbf{R}}^{\prime(\alpha)} \mathbf{V}^{\prime}(\mathbf{x})=-\nabla U^{\prime}\left(\mathbf{x}^{\prime}\right)$, and in the $\mathbf{k}^{\prime}$-space we have $\bar{V}_{l}^{\prime}\left(\mathbf{k}^{\prime}\right)=-\hat{\bar{B}}_{l m}^{\prime(\alpha)} 2 \pi i k_{m}^{\prime} \bar{U}^{\prime}\left(\mathbf{k}^{\prime}\right)$ and $\hat{\bar{R}}_{m l}^{\prime(\alpha)} \bar{V}_{l}^{\prime} \quad\left(\mathbf{k}^{\prime}\right)=-2 \pi i k_{m}^{\prime} \bar{U}^{\prime}\left(\mathbf{k}^{\prime}\right)$. Evidently for $\bar{\Pi}_{l}^{\prime(\alpha)}\left(\mathbf{k}^{\prime}\right)$ we have the linear expression $\bar{\Pi}_{l}^{\prime}\left(\mathbf{k}^{\prime}\right)=-\hat{\bar{B}}_{l m}^{\prime(\alpha)} 2 \pi i k_{m}^{\prime}$, or in x-space $\overline{\mathbf{\Pi}}^{\prime}\left(\mathbf{x}^{\prime}-\mathbf{y}^{\prime}\right)=-\hat{\overline{\mathbf{B}}}^{(\alpha)} \nabla_{x^{\prime}} \delta\left[\mathbf{x}^{\prime}-\mathbf{y}^{\prime}\right]$.

But what if the diagonal tensor $\overline{\mathbf{B}}^{(\alpha)}$ is unknown? Or to put it more precisely, what if we know that there exists some symmetry, but the orientation of the principal axes is unknown and the parameter $\alpha$ is unknown? In this case, we return to Equation (12), which is valid for any stochastically homogeneous, positive definite, random fields $\boldsymbol{\sigma}(\mathbf{x})$, and study the odd vector $\bar{\Pi}_{l}^{\prime}\left(\mathbf{k}^{\prime}\right)$, along with its formal Taylor expansion about $\mathbf{k}^{\prime}=0$ :

$$
\bar{\Pi}_{l}^{\prime}\left(\mathbf{k}^{\prime}\right)=\sum_{n=n_{1}+n_{2}+n_{3}=1}^{\infty} \frac{1}{(n) !} \frac{\partial^{n} \bar{\Pi}_{l}^{\prime}(0)}{\partial k_{1}^{\prime n_{1}} \partial k_{2}^{\prime n_{2}} \partial k_{3}^{\prime n_{3}}} k_{1}^{\prime n_{1}} k_{2}^{\prime n_{2}} k_{3}^{\prime n_{3}}
$$

Inserting linear part of expansion (29) in (12), we can write

$$
\bar{V}_{l}^{\prime}\left(\mathbf{k}^{\prime}\right)=-\bar{B}_{l m}^{\prime} 2 \pi i k_{m}^{\prime} \bar{U}^{\prime}\left(\mathbf{k}^{\prime}\right), \quad \bar{B}_{l m}^{\prime}=-\frac{1}{2 \pi i} \frac{\partial \bar{\Pi}_{l}^{\prime}(0)}{\partial k_{m}^{\prime}}
$$

In this case, we have the averaged equation:

$$
V_{l}^{\prime}\left(\mathbf{x}^{\prime}\right)=-\overline{\mathrm{B}}_{l m}^{\prime} \frac{\partial U^{\prime}\left(\mathbf{x}^{\prime}\right)}{\partial x_{m}^{\prime}}
$$

and in the general case, we have $\bar{B}_{l m}^{\prime}$ - the constant real tensor of the effective conductivity that is symmetric and positive definite. Thus, if we know the components $\bar{\Pi}_{l}^{\prime}\left(\mathbf{k}^{\prime}\right)$, we can find from Equation (30) the effective conductivity tensor $\overline{\mathbf{B}}^{\prime}$ and, by using the standard method, we can find its real eigenvalues and orthogonal eigenvectors. Transition to a new orthogonal system associated with the eigenvectors and transformation of the tensor $\overline{\mathbf{B}}^{\prime}$ to the new coordinates lead to a diagonal tensor $\overline{\mathbf{B}}$, whose components are the eigenvalues for the tensor $\overline{\mathbf{B}}^{\prime}$. For each $\alpha$, the tensor $\overline{\mathbf{B}}(\mathbf{k})$ is unique and reversible, and its components are: $\overline{\mathrm{B}}_{l l}(\mathbf{k})=-\bar{\Pi}_{l}(\mathbf{k}) / 2 \pi i k_{l}$, $\bar{B}_{l m}(\mathbf{k})=0$, if $l \neq m$ (no summation over $l !$ ) 
Even if there is or not any types of global symmetry like the one discussed above, the fact remains that if the stochastically homogeneous field of the local random conductivity tensor $\sigma_{m l}(\mathbf{x})$ is symmetric and elliptic, the constant tensor of the effective conductivity $\bar{B}_{m l}^{\prime}$ is symmetric and elliptic as well. In this general case, the "principal" orthogonal coordinate system exists in which the tensor $\overline{\mathbf{B}}$ is diagonal.

As we showed in Section 5, in the cases of global symmetry, we find the exact, unique, and reversible solution - the diagonal tensor. The difference here lies in the fact that in the case of global symmetry, the components of the diagonal tensor $\overline{\mathbf{B}}(\mathbf{k})$ are dependent on some invariants related to the type of symmetry. It is evident that these tensors are not necessarily elliptic for any k.

\section{ALTERNATIVE APPROACH}

Most of the publications related to the present subject have used a different approach from that discussed in this paper. From the outset, many investigators have attempted to find a basic flow law like relations (algebraic or, more general, operator-related) between the averaged flow velocity vector and the gradient of mean pressure (head). It is pertinent to note again that in general, the transformation of some vector into a different noncollinear vector is non-unique and can be realized with an unbounded set of mapping. Thus, the approach generally leads to an illposed inverse problem. We showed in Section 4, for the general case of a stochastically homogeneous conductivity field, that a more simple and unique exact relation between mean velocity field and mean pressure (head) exists. However, in some cases of global symmetry this relation can be transformed into equations which relate the flow velocity and the gradient of pressure (head). Nevertheless, we will more closely analyze the alternative approach and return to the exactly averaged Equation (12), where the vector $\overline{\mathbf{\Pi}}(\mathbf{k})$ satisfies Equation (13). Now we will analyze the real vector $\boldsymbol{\Gamma}(\mathbf{x})$ as a sum of unique even and odd parts: $\boldsymbol{\Gamma}(\mathbf{x})=\boldsymbol{\Gamma}_{*}(\mathbf{x})+\Gamma^{*}(\mathbf{x})$, where $\boldsymbol{\Gamma}_{*}(\mathbf{x})=[\boldsymbol{\Gamma}(\mathbf{x})+\boldsymbol{\Gamma}(-\mathbf{x})] / 2$ is a real, even function and $\boldsymbol{\Gamma}^{*}(\mathbf{x})=[\boldsymbol{\Gamma}(\mathbf{x})-\boldsymbol{\Gamma}(-\mathbf{x})] / 2$ is a real, odd function. In the general case, $\overline{\boldsymbol{\Gamma}}(\mathbf{k})$ is a unique complex vector function. Because $\bar{G}(\mathbf{k})$ is a real, even function in the same way, we see that in general $\overline{\boldsymbol{\Pi}}(\mathbf{k})=\overline{\boldsymbol{\Pi}}_{*}(\mathbf{k})+\overline{\boldsymbol{\Pi}}^{*}(\mathbf{k})$ is a unique complex vector function, where $\overline{\boldsymbol{\Pi}}_{*}(\mathbf{k})=\overline{\boldsymbol{\Gamma}}_{*}(\mathbf{k}) / \bar{G}(\mathbf{k})$ is real and even and $\overline{\mathbf{\Pi}}^{*}(\mathbf{k})=\overline{\boldsymbol{\Gamma}}^{*}(\mathbf{k}) / \bar{G}(\mathbf{k})$ imaginary and odd.

Now substituting the complex vector $\overline{\mathbf{\Pi}}(\mathbf{k})$ in Equation (13) and comparing the real and imaginary parts from both sides of the equation, we have $2 \pi i \mathbf{k} \overline{\boldsymbol{\Pi}}_{*}(\mathbf{k})=0$. This equation is valid if $\overline{\boldsymbol{\Pi}}_{*}(\mathbf{k})=0$, which is equivalent to $\overline{\boldsymbol{\Pi}}(\mathbf{k})$ being an imaginary odd vector, or if $\overline{\boldsymbol{\Pi}}_{*}(\mathbf{k}) \neq 0$, which means that this vector is orthogonal to the vector $\mathbf{k}$, which, of course, does not mean that the vector $\overline{\boldsymbol{\Pi}}^{*}(\mathbf{k})$ is collinear to $\mathbf{k}$. In both cases the vector $\overline{\boldsymbol{\Pi}}_{*}(\mathbf{k})$ does not affect the operator $2 \pi i \mathbf{k} \overline{\mathbf{\Pi}}(\mathbf{k})$. Thus, for describing $\overline{\mathbf{V}}(\mathbf{k})$ or $\mathbf{V}(\mathbf{x})$ from Equations (12) or (14), we need to use the vector-functions $\overline{\mathbf{\Pi}}(\mathbf{k})$ or $\boldsymbol{\Pi}(\mathbf{x})$ respectively. On the other hand, for finding $\bar{U}(\mathbf{k})=\bar{f}(\mathbf{k}) / 2 \pi i \mathbf{k} \overline{\boldsymbol{\Pi}}^{*}(\mathbf{k})$, we use the vector $\overline{\boldsymbol{\Pi}}^{*}(\mathbf{k})$. 
Now we introduce the real and even tensor $\overline{\mathbf{B}}^{*}(\mathbf{k})$ and imaginary and odd tensor $\overline{\mathbf{B}}_{*}(\mathbf{k})$ that satisfies the equations:

$$
\bar{\Pi}_{l}^{*}(\mathbf{k})=-\overline{\mathrm{B}}_{l m}^{*}(\mathbf{k}) 2 \pi i k_{m}, \quad \bar{\Pi}_{* l}(\mathbf{k})=-\overline{\mathrm{B}}_{* l m}(\mathbf{k}) 2 \pi i k_{m}
$$

If we insert $\overline{\boldsymbol{\Pi}}_{*}(\mathbf{k})$ and $\overline{\boldsymbol{\Pi}}^{*}(\mathbf{k})$ from Equation (32) into (13), we obtain the condition of compatibility for $\overline{\mathbf{B}}^{*}(\mathbf{k})$ and $\overline{\mathbf{B}}_{*}(\mathbf{k})$ :

$4 \pi^{2} \bar{G}(\mathbf{k}) \mathbf{k} \overline{\mathbf{B}}^{*}(\mathbf{k}) \mathbf{k}=1, \mathbf{k} \overline{\mathbf{B}}_{*}(\mathbf{k}) \mathbf{k}=0$

Substitute $\overline{\boldsymbol{\Pi}}_{*}(\mathbf{k})$ and $\overline{\boldsymbol{\Pi}}^{*}(\mathbf{k})$ from Equation (32) into (12), and if the convolution converges, we have:

$\overline{\mathbf{V}}(\mathbf{k})=-\left[\overline{\mathbf{B}}^{*}(\mathbf{k})+\overline{\mathbf{B}}_{*}(\mathbf{k})\right] 2 \pi i \mathbf{k} \bar{U}(\mathbf{k}), \mathbf{V}(\mathbf{x})=-\int\left[\mathbf{B}^{*}(\mathbf{x}-\mathbf{y})+\mathbf{B}_{*}(\mathbf{x}-\mathbf{y})\right] \nabla U(\mathbf{y}) d y^{3}$

Here real tensors $\mathbf{B}^{*}(\mathbf{x})$ and $\mathbf{B}_{*}(\mathbf{x})$ are even and odd respectively.

Let the tensor $\overline{\mathbf{B}}^{*}(\mathbf{k})$ and $\mathbf{B}_{*}(\mathbf{k})$ be symmetric over subscripts, because adding to them any skew-symmetric tensor does not affect the quadratic forms in Equation (33).

The definition of the Fourier transformation $\overline{\mathbf{B}}^{*}(\mathbf{k})$ and $\overline{\mathbf{B}}_{*}(\mathbf{k})$ with the systems of Equations (32) leads to two systems of three linear algebraic equations for each $\mathbf{k}$, with each equation containing three from six unknown components $\bar{B}_{m l}^{*}(\mathbf{k})$ or $\bar{B}_{* l m}(\mathbf{k})$ respectively. In the $x$ space, this problem amounts to two systems containing three differential equations each with six unknown functions - components $B^{*}{ }_{l m}(\mathbf{x})$.

Both systems of Equation (32) are underdetermined and in general have unlimited sets of solutions. It is well known that $\overline{\mathbf{B}}_{\otimes}(\mathbf{k})$, the general solution (all infinite sets of solutions) for a singular non-uniform system of linear algebraic equations, can be presented for each $\mathbf{k}$ as a sum of $\overline{\mathbf{B}}_{0}(\mathbf{k})$, any particular solution of the system, and $\overline{\mathbf{B}}^{\mathrm{e}}(\mathbf{k})$, an infinite set of solutions for the uniform system $\bar{B}_{l m}^{\mathrm{e}}(\mathbf{k}) k_{m}=0$. (The geometric sense of the uniform system is that all three vector-rows for the tensor $\overline{\mathbf{B}}^{\mathrm{e}}(\mathbf{k})$ are orthogonal to the vector $\mathbf{k}$.) For this reason, as indicated by Indelman and Abramovich, (1994), adding any of the solutions $\overline{\mathbf{B}}^{\mathrm{e}}(\mathbf{k})$ to $\overline{\mathbf{B}}_{0}(\mathbf{k})$ in Equation (34) with the known $2 \pi i \mathbf{k} \bar{U}(\mathbf{k})$ does not affect the computation of $\overline{\mathbf{V}}(\mathbf{k})$.

The analyses presented thus far show that the basic model (see Equations (12) and (14)) which realized the relation between the mean flow velocity $\overline{\mathbf{V}}(\mathbf{k})$ or $\mathbf{V}(\mathbf{x})$ and the mean pressure (head) in the general case of a stochastically homogeneous conductivity field-is exact and unique. The relationship between the averaged fields is prescribed with exact and unique operators, vectors $\overline{\boldsymbol{\Pi}}(\mathbf{k})$ or $\boldsymbol{\Pi}(\mathbf{x})$. On the other hand in some cases of global symmetry this model as well lead to linear nonlocal relation between mean flow velocity and gradient of mean pressure (head) prescribed with exact and unique kernel-tensor.

In addition, we examined the more complicated alternative model, which in general case of stochastically homogeneous conductivity random field realized the linear connection between the mean flow velocity and gradient of head. In this case, formally the vector $\overline{\mathbf{\Pi}}_{*}(\mathbf{k})$, the real and even part of $\overline{\mathbf{\Pi}}(\mathbf{k})$, is a product of some imaginary and odd symmetric tensor $\overline{\mathbf{B}}_{*}(\mathbf{k})$ and vector 
$2 \pi i \mathbf{k}$. We also write the imaginary and odd vector $\overline{\boldsymbol{\Pi}}^{*}(\mathbf{k})$ as the product of some real tensor $\overline{\mathbf{B}}^{*}(\mathbf{k})$ and vector $2 \pi i \mathbf{k}$. We showed that problem of deriving tensors $\overline{\mathbf{B}}^{*}(\mathbf{k})$ and $\overline{\mathbf{B}}_{*}(\mathbf{k})$ in general case is ill-posed. Thus, we see that creation of similar complicated models is inefficient.

\section{TWO-DIMENSIONAL STEADY-STATE FLOW IN AN UNBOUNDED DOMAIN}

In Section 2, we briefly discussed the case of two-dimensional flow. Now we will examine this case in more detail. We consider a system of equations that does not explicitly contain $u(\mathbf{x})$ :

$$
\nabla \mathbf{v}(\mathbf{x})=f(\mathbf{x}), \mathbf{v}(\mathbf{x})=\boldsymbol{\sigma}(\mathbf{x}) \mathbf{h}(\mathbf{x}), \nabla \times \mathbf{h}(\mathbf{x})=0
$$

In two-dimensional space, this system includes four scalar independent equations for four scalar functions and is closed. We assume that in an unbounded domain, $h_{l}(\mathbf{x})$ and $v_{l}(\mathbf{x})$ vanish at infinity. It is evident that if the function $f(\mathbf{x})$ is integrable and has bounded support, the system has a unique solution.

Now we introduce an auxiliary system for Green`s velocity $\gamma(\mathbf{x}, \mathbf{y})$ and the intensity $\mathbf{s}(\mathbf{x}, \mathbf{y})$ that vanish at infinity:

$$
\nabla_{x} \gamma(\mathbf{x}, \mathbf{y})=\delta(\mathbf{x}-\mathbf{y}), \gamma(\mathbf{x}, \mathbf{y})=\boldsymbol{\sigma}(\mathbf{x}) \mathbf{s}(\mathbf{x}, \mathbf{y}), \nabla_{x} \times \mathbf{s}(\mathbf{x}, \mathbf{y})=0
$$

and write the solution of the system in (35)

$$
\mathbf{h}(\mathbf{x})=\int \mathbf{s}(\mathbf{x}, \mathbf{y}) f(\mathbf{y}) d y^{2} \quad, \quad \mathbf{v}(\mathbf{x})=\int \gamma(\mathbf{x}, \mathbf{y}) f(\mathbf{y}) d y^{2}
$$

Taking into account that the tensor-field $\boldsymbol{\sigma}(\mathbf{x})$ is stochastically homogeneous, we have, after averaging the equations in (37), the averaged solution of system (35):

$$
\mathbf{H}(\mathbf{x})=\int \mathbf{S}(\mathbf{x}-\mathbf{y}) f(\mathbf{y}) d y^{2}, \mathbf{V}(\mathbf{x})=\int \boldsymbol{\Gamma}(\mathbf{x}-\mathbf{y}) f(\mathbf{y}) d y^{2}
$$

Although the fields $\mathbf{S}(\mathbf{x})$ and $\boldsymbol{\Gamma}(\mathbf{x})$ vanish at infinity, they are not absolute integrable in all space. Nevertheless, the convolutions in Equation (38) converge because the function $f(\mathbf{x})$ has bounded support. Now, using the generalized Fourier transform (9) for Equations (38), we have two relations:

$$
\overline{\mathbf{H}}(\mathbf{k})=\overline{\mathbf{S}}(\mathbf{k}) \bar{f}(\mathbf{k}), \overline{\mathbf{V}}(\mathbf{k})=\overline{\mathbf{\Gamma}}(\mathbf{k}) \bar{f}(\mathbf{k})
$$

It should be noted that from Equations (38) follows the condition $\mathbf{k} \times \overline{\mathbf{S}}(\mathbf{k})=0$; that is, the vector $\overline{\mathbf{S}}(\mathbf{k})$ is collinear to the vector $\mathbf{k}$ and is odd and imaginary.

So, if the mean Green's velocity and the intensity-vector are known, we have a closed system (38), (39) and can for any $f(\mathbf{x})$ solve the direct problem and find the mean velocity and the intensity in $\mathbf{x}$ and $\mathbf{k}$-spaces.

\section{Global symmetric two-dimensional systems}

When the system is globally isotropic, the vectors $\overline{\mathbf{S}}(\mathbf{k})$ and $\overline{\boldsymbol{\Gamma}}(\mathbf{k})$ are odd and imaginary and mutually collinear. In this case, we have:

$$
\overline{\boldsymbol{\Gamma}}(\mathbf{k})=\lambda^{(i)}(k) \overline{\mathbf{S}}(\mathbf{k}), \quad \lambda^{(i)}(k)=\bar{\Gamma}(\mathbf{k}) / \bar{S}(\mathbf{k})
$$


Here $\lambda^{(i)}(k)$ is a real positive scalar function of $k$. Then, similarly, we have:

$\bar{V}_{l}(\mathbf{k})=\bar{B}_{l m}^{(i)}(k) \bar{H}_{m}(\mathbf{k}), B_{l m}^{(i)}(k)=\bar{\lambda}^{(i)}(k) \delta_{l m}$

and if the convolution exists,

$$
V_{l}(\mathbf{x})=\int B_{l m}^{(i)}(\mathbf{x}-\mathbf{y}) H_{m}(\mathbf{y}) d y^{2} \text { or } \int R_{m l}^{(i)}(\mathbf{x}-\mathbf{y}) V_{l}(\mathbf{y}) d y^{2}=H_{m}(\mathbf{x})
$$

When $\bar{f}(\mathbf{k})=1$, we have $\overline{\mathbf{H}}(\mathbf{k})=\overline{\mathbf{S}}(\mathbf{k})$, and from the first averaged equation in (41) we obtain for the isotropic tensors $\overline{\mathbf{R}}(k)$ and $\mathbf{R}(x): \bar{R}_{m l}(k)=2 \pi i k_{r} \bar{S}_{r}(\mathbf{k}) \delta_{m l}, R_{m l}(x)=\nabla \mathbf{S}(\mathbf{x}) \delta_{m l}$.

If an appropriate convolution exists, we can write the averaged nonlocal equation

$$
\int V_{m}(\mathbf{x}-\mathbf{y}) \nabla \mathbf{S}(\mathbf{y}) d y^{2}=H_{m}(\mathbf{x})
$$

Now we rewrite the tensor $\bar{R}_{m l}(k)$ as $\bar{R}_{m l}(k)=2 \pi i S_{r}(\mathbf{k}) \delta_{r p} k_{p} \delta_{m l}$, and receive the convolution that matches Equation (43):

$$
-\int \nabla_{y} V_{m}(\mathbf{x}-\mathbf{y}) \mathbf{S}(\mathbf{y}) d y^{2}=H_{m}(\mathbf{x})
$$

Taking into account the estimation $S_{l}(\mathbf{y}): y_{l} / 2 \pi \sigma_{*} y^{2}$ for small $y$ and the differentiability of the flow velocity $\mathbf{V}(\mathbf{x}-\mathbf{y})$, we see that the integral converges at $\mathbf{y}=0$. For very large $y$ we use the estimate $S_{l}(\mathbf{y}) \sim y_{l} / 2 \pi \sigma^{*} y^{2}$ and $V_{m}(\mathbf{x}-\mathbf{y}) \sim q y_{m} / 2 \pi y^{2}$ for finite $\mathbf{x}$. Thus, we have the estimate for very large $y: S_{l}(\mathbf{y}) \nabla_{y} V_{m}(\mathbf{x}-\mathbf{y}) \sim y_{m} / 2 \pi y^{4} \sigma^{*}$. The integral converges at infinity. The convolutions in Equations (44), (43), and (42) apply.

If the field $\boldsymbol{\sigma}(\mathbf{x})$ is globally orthotropic, for the orthotropically oriented coordinate system, the vector $\overline{\boldsymbol{\Gamma}}(\mathbf{k})$ is the product of the diagonal tensor, whose components are invariant to $\left|k_{1}\right|$, and $\left|k_{2}\right|$ to the vector $\mathbf{k}$. In this case, $\overline{\boldsymbol{\Gamma}}(\mathbf{k})$ is an odd imaginary vector that is in general noncollinear with the vector $\mathbf{k}$, and we can write: $\bar{S}_{l}(\mathbf{k})=\bar{\lambda}_{l}^{s}\left(\left|k_{1}\right|,\left|k_{2}\right|\right) k_{l}, \bar{\Gamma}_{l}(\mathbf{k})=\bar{\lambda}_{l}^{\Gamma}\left(\left|k_{1}\right|,\left|k_{2}\right|\right) k_{l}$ (no summation over 1 !)

Combining again with the latter equation in (39), we find the relationship between the velocity vector and the hydraulic field intensity for the global orthotropic system:

$$
\bar{V}_{l}(\mathbf{k})=\bar{B}_{l m}^{(o)}(\mathbf{k}) \bar{H}_{m}(\mathbf{k}), \bar{B}_{l m}^{(o)}(\mathbf{k})=\bar{\lambda}_{l}^{(o)}\left(\left|k_{1}\right|,\left|k_{2}\right|\right) \delta_{l m}, \quad \bar{\lambda}_{l}^{(o)}=\lambda_{l}^{\Gamma} / \lambda_{l}^{s}
$$

(No summation over $l$ !)

And if the convolutions exist, we can write

$$
V_{l}(\mathbf{x})=\int B_{l m}^{(o)}(\mathbf{x}-\mathbf{y}) H_{m}(\mathbf{y}) d y^{2} \text { or } H_{m}(\mathbf{x})=\int R_{m l}^{(o)}(\mathbf{x}-\mathbf{y}) V_{l}(\mathbf{y}) d y^{2}
$$

Thus, if the two-dimensional system is global symmetric (isotropic or orthotropic), the tensors $\mathbf{B}^{(\alpha)}(\mathbf{k}), \overline{\mathbf{R}}^{(\alpha)}(\mathbf{k})$ in $\mathbf{k}$-space and $\mathbf{B}^{(\alpha)}(\mathbf{x}), \mathbf{R}^{(\alpha)}(\mathbf{x})$ in $\mathbf{x}$-space in the appropriate coordinate systems are diagonal, and therefore, unique. In the homogenization limit, we have the effective conductivity diagonal tensor $\hat{\bar{B}}_{l m}^{(\alpha)}=\bar{\lambda}_{l}^{(\alpha)}(0) \delta_{l m}$, and hence in any orthogonal coordinate system, the local equations are $V_{l}(\mathbf{x})=\hat{\bar{B}}_{l m}^{(\alpha)} H_{m}(\mathbf{x})$ or $H_{m}(\mathbf{x})=\hat{\bar{R}}_{m l}^{(\alpha)} V_{l}(\mathbf{x})$, where the constant tensors $\hat{\overline{\mathbf{B}}}^{(\alpha)}, \hat{\overline{\mathbf{R}}}^{(\alpha)}$ are symmetric and elliptic. 
As we noted in Section 2, if $q=\int f(\mathbf{x}) d x^{2}=0$, the function $u(\mathbf{x})$ vanishes at infinity and is unique. Therefore, we can rewrite equations (46) in the form:

$$
\mathbf{V}(\mathbf{x})=\int \mathbf{B}^{(\alpha)}(\mathbf{x}-\mathbf{y}) \nabla U(\mathbf{y}) d y^{2}, \nabla U(\mathbf{x})=\int \mathbf{R}^{(\alpha)}(\mathbf{x}-\mathbf{y}) \mathbf{V}(\mathbf{y}) d y^{2}
$$

It is easy to see that in the general case (without any symmetry) the linear relations between complex vectors-field $\overline{\mathbf{\Gamma}}(\mathbf{k})$ and $\overline{\mathbf{S}}(\mathbf{k})$, as $\overline{\mathbf{V}}(\mathbf{k})$ and $\overline{\mathbf{H}}(\mathbf{k})$, respectively, can be realized with some tensor $\overline{\mathbf{B}}(\mathbf{k})$. As in the three-dimensional case, the system of linear algebraic equations for finding $\bar{B}_{l m}(\mathbf{k})$ is underdetermined, because for each vector $\mathbf{k}$ we have a system of two equations for three (in the case of indices symmetry) or, in the general case, four components. Thus the problem is ill-posed. On the other hand for the general case without any global symmetry, we can use the averaged equations (38) and (39).

\section{Summary}

We have described the general form for the exactly averaged system of basic equations of flow in a stochastically homogeneous unbounded field with sources. We examined the validity of the averaged descriptions and the generalized law for some nonlocal models. The approach described in the present paper does not require the assumption of existence of any small parameters, for example, small scales of heterogeneity or small perturbation of conductivity field.

For the common case of a three-dimensional stochastically homogeneous conductivity field, a new exactly averaged basic nonlocal equation with unique kernel-vector was presented. We showed the uniqueness and analyzed the properties of the averaged nonlocal equation with kernel-tensor for three types of global symmetry (isotropy, transversal isotropy and orthotropy) in the three-dimensional conductivity field and two types (isotropy and orthotropy) in the twodimensional field.

We analyzed the structure of nonlocal basic equations with a kernel-tensor, for the case in which a global symmetry of the conductivity field does not exist, and showed that this more complicated model leads to an ill-posed inverse problem, and is inefficient.

The intention of the approach presented here is different from other usual approaches. The intention of "other approaches" is to solve the local stochastic equations directly and to find some tensor-operator, that describes the relations between mean flow velocity vector and the gradient of mean head. This approach is very difficult and is inaccurate as we explained in the present paper. In contrast, our intention is to use the existence of appropriate random Green`s functions, find the averaged fields, and the relations between the fields that are independent from the density of sources. This approach leads to exact forms of the averaged unique nonlocal equations.

\section{Acknowledgments}

The authors would like to thank Dr. D. Silin of Lawrence Berkeley National Laboratory for constructive comments. This work was conducted for the U.S. Department of Energy under Contract No. DE-AC02-05CH11231. We greatly appreciate the critical reviews provided by the TIPM reviewers. 


\section{References}

Bakhvalov, N.S., and G.P. Panasenko: 1989, Homogenization: Averaging Processes in Periodic Media; Mathematical Problems in the Mechanics of Composite Material, Kluver Academic, Dordrecht, Boston, MA.

Bonilla, F.A., and Cushman J.H.: 2001, On perturbative expansions to the stochastic flow problem. Transport in Porous Media, 42, 3-35.

Courant, R.: 1962, Partial Differential Equations, Interscience, New York.

Dagan, G.: 1979, The generalization of Darcy`s law for nonuniform flows. Water Resour. Res., 15, (1), 1-7.

Dykhne, A.M.: 1971, Conductivity of two-dimensional two-phase systems. Soviet Physics. JETP, 32 (1), $63-65$.

Hornung, U. (ed.): 1997, Homogenization and Porous Media, IAM, Springer-Verlag, Berlin.

Indelman, P., and Abramovich B.:1994, Nonlocal properties of nonuniform averaged flows in heterogeneous media, Water Resour.Res., 30 (12),3385-3393.

Indelman, P.: 1996, Averaging of unsteady flows in heterogeneous media of stationary conductivity. J. Fluid Mech., 310, 39-60.

Indelman, P.: 2001, Steady-state source flow in heterogeneous porous media. Transport in Porous Media, 45:105-127.

Indelman, P.: 2002, On mathematical models of average flow in heterogeneous formations. Transport in Porous Media, 48:209-224.

King, P.R.: 1987, The use of field theoretic methods for study of flow in a heterogeneous porous media. J. Phys. A, Math., Gen., 20, 3935-3947.

Keller, J.B.: 1964, A theorem on the conductivity of a composite media. J. Math.Phys.: 5: 548549.

Keller, J.B.: 2001, Flow in random porous media. Transport in Porous Media, 43: 395-406.

Lifshitz, I.M., S.A.Grodeskuul, L.A.Pastur .:1988, Introduction to theory of disordered solids, John Wiley, New York.

Lomakin, V.A.: 1970, Statistical problem of mechanics solid deformable media (in Russian), Nauka, Moskow, 77-85.

Matheron, G.: 1967, Elements Pour Une Theorie Des Mileeux Poreux. Masson \& Cie, Editeurs, Paris.

Neuman, S.P., and Orr S.: 1993, Prediction of steady state flow in nonuniform geologic media by conditional moments: exact nonlocal formalism, effective conductivies, and weak approximation, Water Resour. Res., 29 (2), 341-364.

Saffman, P.G.: 1971, On the boundary conditions at the surface of a porous medium, Stud. Appl.Math., 93-101.

Schwartz, L.: 1961, Methods Mathematiques Pour Les Sciences Physiques, Herman \& Cie, Paris. 
Shvidler, M.I.: 1966, Source-solution of the problem of unsteady flow trough random porous medium, Fluid Dyn.1, (4), 95-98.

Shvidler, M.I.:1985, Statistical Hydrodynamics of Porous Media. (In Russian), Nedra, Moscow.

Shvidler, M., and K.Karasaki.: 1999, Investigation of the exactly averaged equations of flow and transport in random porous media. Abstract, In Transactions, AGU Fall Meeting, San Francisco, 1999.

Shvidler, M., and K. Karasaki: 2001, Exactly Averaged Stochastic Equations for Flow and Transport in Random Media. Earth Sciences Division Ernest Orlando Lawrence Laboratory, University of California, Berkeley, LBNL-49529, Preprint, 22.

Shvidler, M., and K.Karasaki: 2005, Averaging of Stochastic Equations for Flow and Transport in Porous Media. Earth Sciences Division, Ernest Orlando Lawrence Berkeley Laboratory, University of California, Berkeley, LBNL-56935, Preprint, 56.

Yosida, K.: 1978, Functional Analysis, Fifth Edition, Springer-Verlag, Berlin.

Zhikov, V.V., S.M. Kozlov, and O.A. Oleinik: 1994, Homogenization of Differential Operators and Integral Functionals, Springer-Verlag, Berlin. 